ARTICLE

Received 15 Jul 2016 | Accepted 4 Apr 2017 | Published 31 May 2017 DOl: 10.1038/ncomms15491 OPEN

\title{
Giant electron-hole transport asymmetry in ultra-short quantum transistors
}

\author{
A.C. McRae ${ }^{1}$, V. Tayari ${ }^{1}$, J.M. Porter ${ }^{1} \&$ A.R. Champagne ${ }^{1}$
}

Making use of bipolar transport in single-wall carbon nanotube quantum transistors would permit a single device to operate as both a quantum dot and a ballistic conductor or as two quantum dots with different charging energies. Here we report ultra-clean 10 to $100 \mathrm{~nm}$ scale suspended nanotube transistors with a large electron-hole transport asymmetry. The devices consist of naked nanotube channels contacted with sections of tube under annealed gold. The annealed gold acts as an $n$-doping top gate, allowing coherent quantum transport, and can create nanometre-sharp barriers. These tunnel barriers define a single quantum dot whose charging energies to add an electron or a hole are vastly different ( $e-h$ charging energy asymmetry). We parameterize the $e-h$ transport asymmetry by the ratio of the hole and electron charging energies $\eta_{\mathrm{e}}-\mathrm{h}$. This asymmetry is maximized for short channels and small band gap tubes. In a small band gap device, we demonstrate the fabrication of a dual functionality quantum device acting as a quantum dot for holes and a much longer quantum bus for electrons. In a $14 \mathrm{~nm}$-long channel, $\eta_{\mathrm{e}-\mathrm{h}}$ reaches up to 2.6 for a device with a band gap of $270 \mathrm{meV}$. The charging energies in this device exceed $100 \mathrm{meV}$.

\footnotetext{
${ }^{1}$ Department of Physics, Concordia University, 7141 Sherbrooke Street West, Montréal, Québec, Canada H4B 1R6. Correspondence and requests for materials should be addressed to A.R.C. (email: a.champagne@concordia.ca).
} 
A $\mathrm{n}$ important feature of ultra-clean (intrinsic) single-walled carbon nanotube (SWCNT) channels is that a small gate voltage can tune them from $n$-type (electron-doped) to $p$-type (hole-doped) devices. A large electron-hole $(e-h)$ transport asymmetry would enable dual functionality quantum SWCNT transistors with drastically different characteristics under electron or hole doping. Unfortunately, the intrinsic transport properties of SWCNTs are mostly $e-h$ symmetric ${ }^{1,2}$ and thus the two types of transport are redundant. Nevertheless, quantum SWCNT devices can be engineered to create an $e-h$ transport asymmetry. This is achieved for instance in SWCNT quantum dot (QD) transistors whose tunnel barrier heights depend on whether the channel is $n$ - or $p$-doped. Such a transport asymmetry has been demonstrated in ultra-clean devices with channel lengths ranging from a few hundreds to $100 \mathrm{~nm}^{3,4}$. Downsizing ultra-clean $e-h$ asymmetric SWCNT transistors to $10 \mathrm{~nm}$ would create QDs, which can be toggled between two vastly different charging energies. They would be useful to explore fundamental nano-electro-mechanical system (NEMS) physics ${ }^{5-9}$ and qubits $2,10-12$ at energy scales close the ones in single molecules ${ }^{13,14}$ or to create THz bolometers ${ }^{15}$, which are sensitive to two different wavelength ranges (one for each $E_{\mathrm{C}}$ ). In addition, a large $e-h$ transport asymmetry in SWCNT transistors could allow them to act as both active logic elements (QDs) for hole doping and interconnects (quantum bus) for electron doping. This dual functionality is needed to create reconfigurable logic circuits which can be programmed with gate voltages ${ }^{16}$ and would support the development of commercial SWCNT electronics ${ }^{17}$.

Here we demonstrate the capability to engineer a giant $e-h$ charging energy and conductance asymmetry in ultra-clean (single QD or ballistic) suspended SWCNT channels whose lengths range down to $14 \mathrm{~nm}$. Ultra-clean SWCNTs used to explore many-body physics and quantum bits have previously been limited to channel lengths above $100 \mathrm{~nm}^{3,7,10-12,18-20}$. The key feature of our fabrication is to use an annealed-gold film as an electrostatic gate directly deposited on a SWCNT (no dielectric spacer). This $n$-dopes the gold-covered SWCNT sections, which then act as contacts to the naked channel. These gold gates create extremely sharp barriers at the contact-to-channel interfaces (few nanometres wide) and these barriers have different heights whether the channel is $n$ - or $p$-doped. We measured transport in five devices under both electron and hole doping of their channels, and at temperatures ranging from 1.3 to $295 \mathrm{~K}$. In a small gap SWCNT device (Device A), $E_{\mathrm{C}}$ 's for electrons and holes differ by orders of magnitude. When this device's channel is $p$ doped, it forms a $102 \mathrm{~nm}$-long low-disorder QD. When the channel is $n$-doped, the device becomes a one-dimensional waveguide where carriers can travel $\approx 330 \mathrm{~nm}$ without losing their phase coherence. In the four devices whose band gaps $E_{\mathrm{g}} \gtrsim 200 \mathrm{meV}$, the charging energy asymmetry ratio $\eta_{\mathrm{e}-\mathrm{h}}$ of the charging energies for holes, $E_{\mathrm{C}}^{\mathrm{h}}$, and electrons, $E_{\mathrm{C}}^{\mathrm{e}}$ ranges from 1.5 to 2.6. We demonstrate that when we bias appropriately these QDs, a modest gate voltage switches their charge transport from $\mathrm{QD}$ to quasi-ballistic. We show that the $e-h$ charging asymmetry scales inversely with channel length and the SWCNT's band gap. The giant $e-h$ asymmetry allows us to very significantly increase $E_{\mathrm{C}}^{\mathrm{h}}$, while reducing $E_{\mathrm{C}}^{\mathrm{e}}$, for a given channel length and band gap. The charging energies of our shortest device (Device B) exceed $100 \mathrm{meV}$, demonstrating the potential for room temperature operation.

\section{Results}

Ultra-short suspended SWCNT-QDs with ballistic contacts. In Table 1, we list for the five studied devices, the following key parameters: band gap $E_{\mathrm{g}}$ as extracted via QD transport, channel length $L_{\mathrm{SEM}}$ measured via scanning electron microscope (SEM) imaging, channel length $L_{\mathrm{G}}$ extracted from QD transport data and ratio $\eta_{\mathrm{e}-\mathrm{h}}$ of the charging energies for holes, $E_{\mathrm{C}}^{\mathrm{h}}$, and electrons, $E_{\mathrm{C}}^{\mathrm{e}}$. Figure 1 summarizes the fabrication and contact geometry of our suspended SWCNT devices ${ }^{5,21}$. We first fabricated suspended gold-on-SWCNT breakjunctions (see Methods). Figure 1a shows a breakjunction and $L_{\text {sus }}$ indicates the length over which the gold is suspended. The substrate (blue) is used as a global back-gate electrode. The final fabrication step is to create a nanometre-long naked SWCNT channel in the centre of the gold breakjunction. To do so, we used a previously reported feedback-controlled electromigration (EM) procedure ${ }^{5,22}$ summarized in Fig. $1 \mathrm{~b}$ (see Methods), where a bias voltage, $V_{\mathrm{B}}$, is applied between the source and drain gold electrodes. This EM process exposes a short naked SWCNT channel. Figure 1c shows top-view SEM images of Devices A and B whose channel lengths are $111 \pm 5$ and $14 \pm 3 \mathrm{~nm}$, respectively. Figure 1d shows the current-gate voltage, $I-V_{\mathrm{G}}$, characteristics at $T=1.3 \mathrm{~K}$ for Devices A (black) and B (red). The EM not only uncovers short naked SWCNT channels but also anneals them such that the QDs in the channels are nearly undoped ${ }^{5}$ with their charge neutrality point close to $V_{G}=0$. Close inspection of the gold surface (Supplementary Fig. 1) reveals that the suspended portion of the gold film changes texture after the EM. The inset of Fig. 1d shows the Fermi level position in the channel under electron or hole doping. The current (conductance) is much higher when the back gate $n$-dopes rather than $p$-dopes the channel. This is consistent with the suspended gold film acting as a top gate which n-dopes the SWCNT sections it covers, and that these nanotube sections act as contacts to the channel (see Fig. 1e). We emphasize that we use pure gold films, without any adhesion layer, to create clean SWCNT-Au interfaces. Gold's workfunction is very close to the threshold, $\approx 5.4 \mathrm{eV}$, where physisorbed metal films switch from $n$-doping to $p$-doping graphene $^{23}$ and SWCNTs ${ }^{24,25}$. A small change in gold's workfunction due to the removing of adsorbed oxygen on the gold during the EM can tune the gold films from $p$ - to $n$ doping 24,26 . We observed this effect in Device B (Supplementary Fig. 2). We note that the fabrication process of our ultra-clean SWCNT devices is compatible with the optical measurement of the SWCNT chirality ${ }^{27,28}$ before the gold deposition. Such ultra-clean SWCNT transistors with a known tube chirality could lead to breakthroughs in SWCNT physics ${ }^{2}$.

The electronic interactions between an ultra-clean SWCNT and gold are weak ${ }^{29}$, and the density of states in the SWCNT is unaffected by the gold film. This is because SWCNT electronic wavefunctions have large wavevectors and the conservation of momentum suppresses the tunnelling matrix elements between these high-momentum states and gold states ${ }^{30}$. It follows that the injection length for an electron to tunnel between gold and the SWCNT is very long $(\mu \mathrm{m})^{29,31}$. Electrostatic disorder leads to stronger SWCNT-gold interactions and shorter injection length ${ }^{29,30}$. This knowledge is necessary to describe the electron transport in the gold covered sections of our SWCNTs and the device design shown in Fig. 1e. The gold film covering the SWCNT sections (1) is thermally anchored to the substrate and not annealed during the EM process. This leaves the sections (1) acting as diffusive (disordered) contacts where the gold-SWCNT coupling is stronger ${ }^{29}$ and where most of the electrons are injected from gold into the SWCNT ${ }^{19,32-34}$. The suspended gold film covering the tube sections labelled (2) is annealed as evidenced from both transport data (Fig. 1d) and the film texture (Supplementary Fig. 1). Finally, the naked SWCNT channel 
Table 1 | Key parameters for the five SWCNT-QD transistors reported.

\begin{tabular}{|c|c|c|c|c|}
\hline Device & $E_{\mathrm{g}}(\mathrm{meV})$ & $L_{\text {SEM }}(\mathrm{nm})$ & $L_{\mathrm{G}}(\mathrm{nm})$ & $\eta_{\mathbf{e}-\mathbf{h}}$ \\
\hline A & $28 \pm 5$ & $111 \pm 5$ & $102 \pm 5$ & Above 100 \\
\hline$B$ & $270 \pm 50$ & $14 \pm 3$ & $7 \pm 5$ & 2.6 \\
\hline C & $190 \pm 20$ & $42 \pm 7$ & $46 \pm 8$ & 1.5 \\
\hline$D$ & $250 \pm 20$ & $16 \pm 4$ & $13 \pm 5$ & 2.1 \\
\hline$E$ & $170 \pm 50$ & $24 \pm 8$ & $15 \pm 5$ & 2.5 \\
\hline
\end{tabular}

a

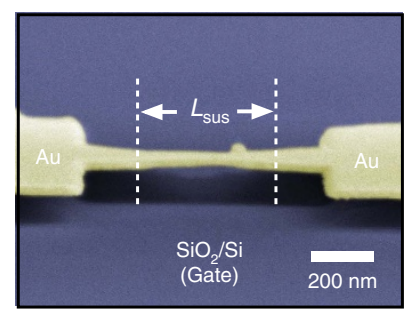

b

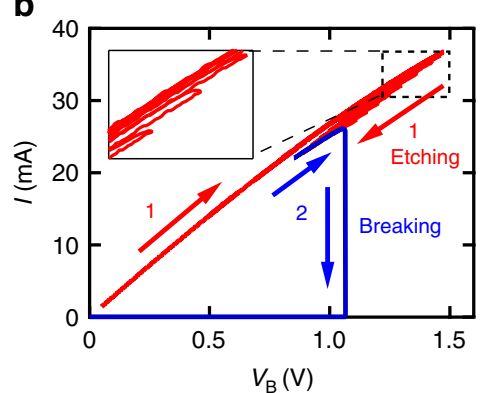

d

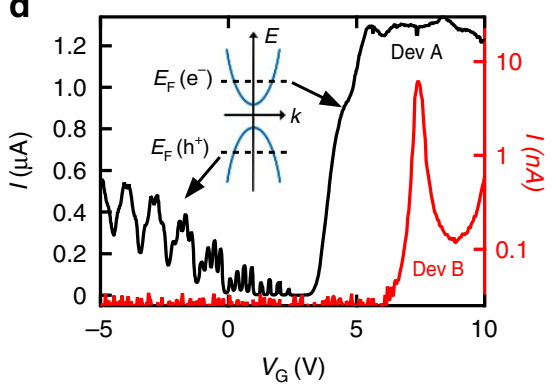

e

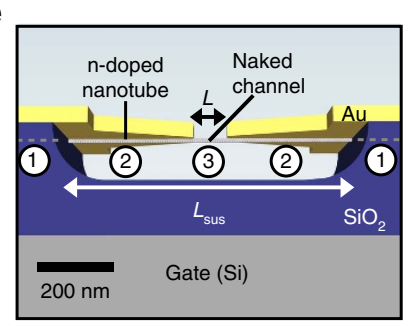

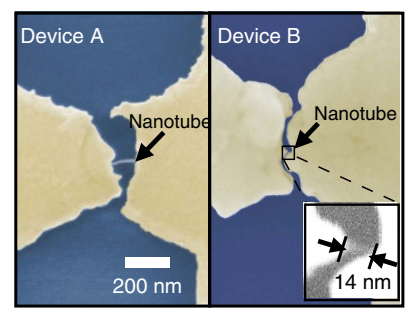

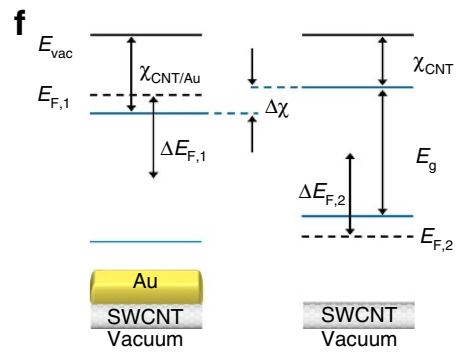

Figure 1 | Ultra-short suspended nanotube QD transistors. (a) Tilted SEM image showing a suspended gold breakjunction fabricated on top of a SWCNT. The breakjunction is suspended over a length $L_{\text {sus }} \approx 350 \mathrm{~nm}$. The back plane (blue) is used as a global back gate. (b) The $I-V_{B} E M$ data for a gold-onSWCNT breakjunction at $T=4 \mathrm{~K}$, showing the process to (1) narrow the junction and (2) create a naked nanotube channel. (c) SEM images of Devices $A$ and $B$ after $E M$. The naked channels are visible and their lengths are $L_{S E M}=111$ and $14 \mathrm{~nm}$, respectively. (d) $I-V_{G}$ transistor data from Devices $A$ (black) and $B(r e d)$, at $T=1.3 \mathrm{~K}$ and $V_{B}=10 \mathrm{mV}$. We measured a much higher conductance for positive $V_{\mathrm{G}}$. This indicates that the suspended gold, annealed during the EM, $n$-dopes the underlying tube. (e) Geometry of our suspended SWCNT transistors. The labels 1, 2 and 3 refer respectively to the disordered onsubstrate nanotube sections, the $n$-doped gold-covered suspended SWCNT sections and the naked SWCNT channel. (f) The electronic bands in the goldcovered (left) and naked (right) suspended tube sections. The quantity $E_{\mathrm{g}}$ is the band gap. The quantities $E_{\mathrm{F}, 1}, E_{\mathrm{F}, 2}, \Delta E_{\mathrm{F}, 1}$ and $\Delta E_{\mathrm{F}, 2}$ are the Fermi energies and the Fermi energy shifts from the center of the band gaps in the gold-covered and naked nanotube, respectively. $\chi_{\mathrm{CNT} / \mathrm{Au}}$ and $\chi_{\mathrm{CN} T}$ are the electron affinities in the gold-covered and naked sections, whereas $\Delta \chi$ is the electron affinity difference between the two.

labelled (3) is thoroughly annealed by the EM process ${ }^{5,21}$ and can be doped with either holes or electrons via $V_{\mathrm{G}}$. The data presented below will demonstrate that the charge transport is ballistic in both sections (2) and (3) of the SWCNTs, and that $e-h$ asymmetric tunnel barriers form where the two sections connect.

The left hand side of Fig. If shows an example of the band diagram for the SWCNT contacts (sections (2) and the right hand side shows the bands in the naked channel (section (3). The dashed lines indicate the positions of the Fermi energies $E_{\mathrm{F}, 1}$ and $E_{\mathrm{F}, 2}$ in each section and their shifts $\Delta E_{\mathrm{F}, 1}$ and $\Delta E_{\mathrm{F}, 2}$ away from the centre of the band gap. The band gap has the same value in both SWCNT sections, as they belong to the same tube. The nanotube's electron affinity, $\chi_{\mathrm{CNT}}$, is defined as the energy between the bottom of the conduction band and the vacuum energy, $E_{\mathrm{vac}}$. On the left side of Fig. 1f, gold transfers charges to the SWCNT and moves $E_{F, 1}$ up from the centre of the gap. The exact amount of doping varies depending on the crystalline orientation of the gold, as well as the quality of annealing (oxygen content). The relevant values of $\Delta E_{\mathrm{F}, 1}$ for our devices are reported to range from 0.05 to $0.2 \mathrm{eV}^{24,25,31,35,36}$ and we use a median value of $0.12 \mathrm{eV}$ to draw our band diagrams. The gold film can also modify the nanotube's electron affinity by $\Delta \chi$. This shift is expected to be $0.03-0.05 \mathrm{eV}^{25,35}$ and we use $0.05 \mathrm{eV}$ to draw the bands in our devices. These parameters correctly predict several features of the data below, such as the presence or absence of a barrier, the sign of the $e-h$ charging energy asymmetry and correlate with the magnitude of the asymmetry. Moreover, the discussion and conclusions below remain valid over the range of reported $\Delta E_{F, 1}$ and $\Delta \chi$ values. The Fermi energy of the naked SWCNT channel, $E_{\mathrm{F}, 2}$, can be tuned using $V_{\mathrm{G}}$. The gating efficiency of the back gate (dielectric of $130 \mathrm{~nm}$ vacuum plus $170 \mathrm{~nm} \mathrm{SiO} 2$ ) is much weaker than the gating efficiency of the gold film (top gate), which is only $3 \AA$ away from the SWCNT ${ }^{25}$. This means that the back gate does not significantly affect $E_{\mathrm{F}, 1}$, but only effectively tunes $E_{\mathrm{F}, 2}$. This is confirmed by the transport data 
a

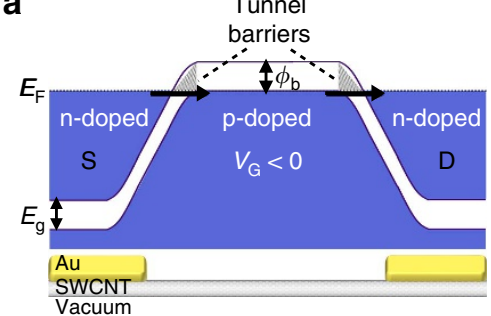

b

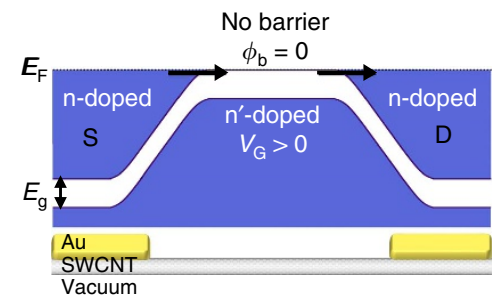

C

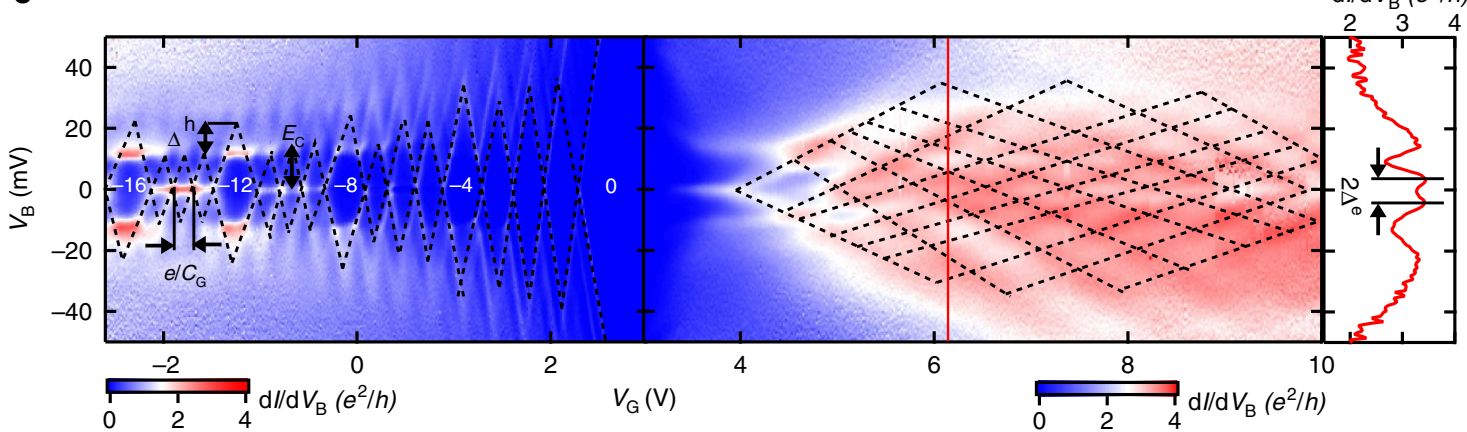

Figure 2 | The $\mathbf{e}-\boldsymbol{h}$ transport asymmetry and ballistic contacts. $(\mathbf{a}, \mathbf{b})$ Schematics of the bands at the junctions between the $n$-doped (gold-covered) nanotube contacts and naked channel in Device $A\left(E_{g}=28 \mathrm{meV}\right.$ ) when the channel is (a) $p$-doped and (b) $n$-doped. Band-to-band tunnel barriers (shaded triangles) of height $\phi_{B}=E_{g}$ form between the nanotube contacts and the $p$-doped channel. On the other hand, when the naked channel is $n$-doped $\phi_{B}=0$. (c) Charge transport data $\left(d l / d V_{B}-V_{B}-V_{G}\right)$ for Device $A$ at $T=1.3 \mathrm{~K}$. The charge neutrality point of the channel is clearly visible around $V_{G}=3 V_{\text {. }} A$ striking asymmetry is visible in the transport data between hole doping $\left(V_{G}<3 \mathrm{~V}\right)$ and electron doping $\left(V_{G}>3 V\right)$ of the channel. For hole doping, a fourfold Coulomb diamond structure indicates a single QD. The gate-to-QD capacitance $C_{G}$ corresponds to a channel length of $102 \pm 5 \mathrm{~nm}$, closely matching $L_{S E M}$ in Fig. 1c. Under electron doping, the transport data show clear Fabry-Pérot quantum interferences (see vertical outset). The spacing between conductance maxima in $V_{B}$ is $5 \pm 1 \mathrm{mV}$, giving $L_{F P}=330 \pm 70 \mathrm{~nm}$. This matches the suspension length of the gold film $L_{\text {sus }}=350 \pm 70 \mathrm{~nm}$ (Supplementary Fig. 1). It confirms that the transport in the suspended gold-covered nanotube sections is ballistic and preserves the quantum phase of the electrons travelling through the channel.

below showing that the lengths (confinement) of the SWCNTQDs are independent of $V_{\mathrm{G}}$. At the junctions between the tube sections (2) and (3), the band diagrams in Fig. If are brought into contact and equilibrate to form homojunctions.

Figure 2a,b show schematics of the homojunctions (Supplementary Note 4 ) in Device A, $E_{\mathrm{g}}=28 \mathrm{meV}$. Figure $2 \mathrm{a}$ shows the band alignment when the channel is hole doped, whereas Fig. $2 b$ shows the electron doping configuration. Tunnel barriers form at the contacts when the channel is $p$-doped. The transport mechanism across the barriers in Fig. $2 \mathrm{a}$ is band-toband tunnelling and has been previously described for SWCNT transistors ${ }^{3,37}$. Approximating the shape of the bands at the homojunctions using the Wentzel-Kramers Brillouin (WKB) model $^{38}$ (shaded triangular regions), the tunnel barrier height is $\phi_{\mathrm{B}}=E_{\mathrm{g}}=28 \mathrm{meV}$. When the channel is electron doped (Fig. 2b), the charge transport only involves the conduction band and there is no tunnel barrier. Low-temperature $(T=1.3 \mathrm{~K})$ transport data from Device A are shown in Fig. 2c, where the colour scales show the differential conductance $d I / d V_{\mathrm{B}}$ as a function of $V_{\mathrm{G}}$ and bias voltage $V_{\mathrm{B}}$. The negative (positive) integer labels $N$ indicate the number of holes (electrons) in the SWCNT channel. Under holedoping of the channel, clear Coulomb blockade diamonds indicate QD transport. When the channel is electron doped, transport data along the $V_{\mathrm{G}}$ and $V_{\mathrm{B}}$ directions show strong FabryPérot interferences characteristic of ballistic transport ${ }^{32,34}$. The dramatic asymmetry in quantum transport between holes (QD) and electrons (ballistic channel) is a consequence of the different contact barriers for the two types of doping. A closer look at the data in Fig. 2c will confirm the device geometry shown in Fig. 1e.

On the left hand side of Fig. 2c, we observe four-fold quantum degeneracy ${ }^{2}$ of the hole-doped QD energy levels. We extract an energy level spacing for holes of $\Delta^{h} \approx 12 \pm 1 \mathrm{meV}$ (height difference between neighbouring tall and short diamonds) and a charging energy $E_{\mathrm{C}}^{\mathrm{h}}=11 \pm 1 \mathrm{meV}$ (height of short diamonds). The width of the Coulomb diamonds for holes is $0.21 \pm 0.01 \mathrm{~V}=e / C_{\mathrm{G}}$, where $C_{\mathrm{G}}$ is the capacitance between the $\mathrm{QD}$ and back gate. We obtain a QD length, $L_{\mathrm{G}}=102 \pm 5 \mathrm{~nm}$, from the measured $C_{\mathrm{G}}$ using a wire over a plane capacitor model (Supplementary Note 3). This length closely matches the length of the channel as measured by SEM, $L_{\mathrm{SEM}}=111 \pm 5 \mathrm{~nm}$. We note that on average in our samples $L_{\mathrm{G}}<L_{\mathrm{SEM}}$ is expected due to the two finite width $p-n$ or $n-n$ ' junctions at each end of the QD. Comparing the measurements of $L_{\mathrm{G}}$ and $L_{\mathrm{SEM}}$ in all devices (Table 1), we find an average $p-n$ junction length of $L_{\mathrm{pn}}=3 \pm 1 \mathrm{~nm}$. An independent estimate of $L_{\mathrm{pn}}$ is obtained from the SWCNT screening length ${ }^{29}, \lambda=\sqrt{\left(\epsilon_{\mathrm{CNT}} / \epsilon_{\mathrm{vac}}\right) d_{\mathrm{CNT}} d_{\mathrm{vac}}}$, where $\epsilon_{\mathrm{CNT}} \approx 10$ is the SWCNT permittivity ${ }^{39}, d_{\mathrm{CNT}} \sim 1.3 \mathrm{~nm}$ is the tube diameter, $\epsilon_{v a c}=1$ is the permittivity of vacuum and $d_{\text {vac }} \approx 0.3 \mathrm{~nm}^{25,40}$ is the thickness of the vacuum dielectric between the tube and gold. This gives $\lambda \approx 2 \mathrm{~nm}$, matching the $L_{\text {pn }}$ extracted above. It is remarkable that we can think of the suspended (annealed) gold film as a gate electrode for the SWCNT, with a dielectric spacer of $3 \AA$, offering the possibility to create extremely sharp $p-n$ junctions. This capability will find applications in testing the ultimate downscaling of SWCNT transistors $^{21,41}$ and creating nanometre-sized phase coherent electronic devices ${ }^{2,12,42,43}$ and NEMS ${ }^{5-9}$.

Although the suspended gold gates in Device A confine holes to a $\mathrm{QD}$ of length $L_{\mathrm{G}}=102 \mathrm{~nm}$, they do not confine electrons inside the naked channel. Using the transport data in the vertical line cut of Fig. 2c, we measure an energy level spacing $\Delta^{\mathrm{e}}=e \Delta V_{\mathrm{B}}=5 \pm 1 \mathrm{meV}$ between the interference maxima. We extract the length of the electron cavity as $L_{\mathrm{FP}}=h v_{\mathrm{F}} /$ $\left(2 \Delta^{\mathrm{e}}\right)=330 \pm 70 \mathrm{~nm}$, where $v_{\mathrm{F}}=8 \times 10^{5} \mathrm{~ms}^{-1}$ is the Fermi 
velocity. This cavity length matches the suspension length of the gold gates, $L_{\text {sus }} \approx 350 \pm 70 \mathrm{~nm}$ (Fig. 1e and Supplementary Fig. 1). The coherent Fabry-Pérot (FP) interferences and cavity length confirm that the majority of the current in the contact sections (2) flows through the SWCNT and not the gold film. This is expected due to the long charge injection length in ultra-clean SWCNTS ${ }^{29,31,40}$. The FP interferences also imply that the annealed gold covering sections (2) does not prevent ballistic transport in the underlying SWCNT ${ }^{44}$ and we do not detect any backscattering at the interfaces between sections (2) and (3). Thus, the naked channel is very nearly ballistic and we extract a very conservative upper bound for its charging energy from the longer FP cavity. Using an open QD model ${ }^{34}$ for the cavity formed by sections 2 and 3 together, we extract an approximate electron charging energy of $E_{\mathrm{C}}^{\mathrm{e}} \sim 0.1 \mathrm{meV}$, giving a charging energy asymmetry ratio $\eta_{\mathrm{e}-\mathrm{h}}=E_{\mathrm{C}}^{\mathrm{h}} / E_{\mathrm{C}}^{\mathrm{e}} \gtrsim 100$. We note that previously reported SWCNT devices, which showed QD to FP $e-h$ asymmetry ${ }^{19,33,45}$ were not fabricated with suspended and annealed gold films, and thus had equal channel lengths for holes and electrons. The important new information in Fig. $2 \mathrm{c}$ are that the annealed gold film acts as a local top gate creating ballistic SWCNT contacts and a gate programmable QD-toballistic transistor with vastly different channel lengths under electron and hole doping. Specifically, applying a small $V_{\mathrm{G}}$ to Device A toggles between a $102 \mathrm{~nm}$ QD and a $330 \mathrm{~nm}$ ballistic wire. In the following section, we demonstrate in SWCNT-QD transistors, whose $L_{\mathrm{G}}$ ranges down to $7 \mathrm{~nm}$ and $E_{\mathrm{g}}$ up to $270 \mathrm{meV}$, that a large $e-h$ transport asymmetry creates QDs with two $E_{\mathrm{C}}$ 's.

Giant $e-h$ transport asymmetry in $200 \mathrm{meV}$ band gap SWCNT-QDs. For room-temperature applications and to explore many-body QD physics, we would like to extend the ability to create large $e-h$ transport asymmetry to SWCNT transistors whose $E_{\mathrm{g}}$ 's are larger than in Device $A$. We studied four larger $E_{\mathrm{g}}$ devices whose naked channels host single QDs whether they are doped with holes or electrons. The heights of the QD contact barriers for the two doping configurations are vastly different and lead to two different $E_{C}$ 's.

Figure $3 \mathrm{a}, \mathrm{b}$ show the homojunctions forming at the interfaces between the contacts and channel in Device C. When the channel is hole doped (Fig. 3a), tall tunnel barriers of $\phi_{\mathrm{B}} \approx E_{\mathrm{g}}=190 \mathrm{meV}$ form where the contacts (sections (2) meet the SWCNT channel (sections (3)). Figure $3 \mathrm{~b}$ shows the configuration when the channel is electron doped. The transport across the $n-n$ ' junctions only involves the conduction band, and the barriers are much smaller, $\phi_{\mathrm{B}} \approx 12 \mathrm{meV}$. This $e-h$ barrier height asymmetry creates an $e-h$ asymmetry of the source-QD and drain-QD capacitances, $C_{\mathrm{S}}$ and $C_{\mathrm{D}}$, respectively. It follows that the $\mathrm{QD}$ charging energy $E_{\mathrm{C}}=e /\left(C_{\mathrm{S}}+C_{\mathrm{D}}+C_{\mathrm{G}}\right)=e / C_{\Sigma}$, depends on whether the $\mathrm{QD}$ is populated with holes or electrons. This is visible in Fig. $3 \mathrm{c}-\mathrm{e}$ where $d I / d V_{\mathrm{B}}-V_{\mathrm{B}}-V_{\mathrm{G}}$ data for Devices $\mathrm{C}, \mathrm{D}$ and $\mathrm{E}$ are shown. The Coulomb diamond heights in Fig. $3 \mathrm{c}-\mathrm{e}$, that is, the addition energies $E_{\text {add }}=E_{\mathrm{C}}+\Delta$, are much larger for holes (left) than for electrons (right).

We use the data in Fig. 3 to quantify both the $e-h$ charging energy asymmetry $\Delta E_{\mathrm{C}}^{\mathrm{e}-\mathrm{h}}=E_{\mathrm{C}}^{\mathrm{h}}-E_{\mathrm{C}}^{\mathrm{e}}$ and the asymmetry ratio $\eta_{\mathrm{e}-\mathrm{h}}=E_{\mathrm{C}}^{\mathrm{h}} / E_{\mathrm{C}}^{\mathrm{e}}$. We extract from Fig. $3 \mathrm{c}-\mathrm{e}$ the heights of the diamonds as a function of the charge occupation number $N$ as shown in Fig. $3 \mathrm{f}-\mathrm{h}$. The dashed lines are interpolations of the odd- $N$ data points, for which $E_{\mathrm{add}}=E_{\mathrm{C}}$, as Devices $\mathrm{C}, \mathrm{D}$ and $\mathrm{E}$ show a twofold degenerate energy spectrum. We note that $\Delta E_{\mathrm{C}}^{\mathrm{e}-\mathrm{h}}$ converges to a roughly constant value at large $N$. To compare the asymmetry between devices, we extract $\eta_{\mathrm{e}-\mathrm{h}}$ at $N=5$. The $\eta_{\mathrm{e}-\mathrm{h}}$ in Devices C, D and $\mathrm{E}$ are $1.5,2.1$ and 2.5 , respectively (Supplementary Note 5). Although the $E_{\mathrm{g}}$ for all three of these devices are comparable, the channels in Devices D and $\mathrm{E}$ are three times shorter than in Device C. Both Devices D and E have significantly larger $\eta_{\mathrm{e}-\mathrm{h}}$ than Device $\mathrm{C}$ and this correlation is also confirmed by Device B (Supplementary Note 2). We thus conclude that $\eta_{\mathrm{e}-\mathrm{h}}$ scales inversely with length. This explains why we can observe large $\eta_{\mathrm{e}-\mathrm{h}}$ in ultra-short QDs whose band gaps range up to $270 \mathrm{meV}$, while previous experiments ${ }^{1}$ on much longer QD devices (few $100 \mathrm{~s}$ of $\mathrm{nm}$ ) showed $\eta_{\mathrm{e}-\mathrm{h}} \approx 1$ in devices with similar band gaps. Figure $3 \mathrm{i}$ shows $I-V_{\mathrm{G}}$ data for Device $\mathrm{E}$ at $V_{B}=40 \mathrm{meV}$. We observe that similarly to Device A (Fig. 2c), it is possible to toggle this large band gap, $E_{\mathrm{g}}=170 \mathrm{meV}$, and very short, $L_{\mathrm{G}}=15 \mathrm{~nm}$, device from behaving as a QD to a nearly ballistic bus by applying modest $V_{\mathrm{G}}$. To further understand the length dependence of $\eta_{\mathrm{e}-\mathrm{h}}$, it is useful to analyse the capacitances of the SWCNT-QDs versus $L_{\mathrm{G}}$.

\section{Discussion}

In Fig. 4a,b, the data in black (red) are for hole (electron)-doped channels. Figure $4 \mathrm{a}$ shows $C_{\mathrm{G}}$ extracted from the widths of the $N=5$ Coulomb diamonds for all devices versus $L_{\mathrm{G}}$ (except for Device $\mathrm{B}$, where only $N=1$ is available). $C_{\mathrm{G}}$ is the same for hole or electron occupations of the $\mathrm{QD}$ and ranges from $\approx 0.05$ to $0.6 \mathrm{aF}$. As expected, $C_{\mathrm{G}}$ scales linearly with $L_{\mathrm{G}}$. Figure $4 \mathrm{~b}$ shows the total QD capacitance $C_{\Sigma}=C_{\mathrm{S}}+C_{\mathrm{D}}+C_{\mathrm{G}}$ extracted from the slopes of the diamonds in Figs 2 and 3 as a function of $L_{\mathrm{G}}$ (Supplementary Note 2). There is a marked $e-h$ asymmetry between the total capacitances for holes and electrons, which we label $\Delta C_{\Sigma}^{\mathrm{e}-\mathrm{h}}=C_{\Sigma}^{\mathrm{e}}-C_{\Sigma}^{\mathrm{h}}$. Figure $4 \mathrm{c}$ shows that both the relative capacitance asymmetry $\Delta C_{\Sigma}^{\mathrm{e}-\mathrm{h}} / C_{\Sigma}^{\mathrm{h}}$ (left axis) and the resulting $\eta_{\mathrm{e}-\mathrm{h}}$ (right axis) decrease rapidly with $L_{\mathrm{G}}$ for Devices B, C, D and $\mathrm{E}$, which all have similar band gaps.

We can also isolate the qualitative effect of $E_{\mathrm{g}}$ on $\eta_{\mathrm{e}-\mathrm{h}}$. A length change of a factor of three between Devices C, D and $E$ (similar $E_{\mathrm{g}}$ 's) changes $\eta_{\mathrm{e}-\mathrm{h}}$ by less than a factor of 2 . An even smaller length difference between Devices A (Fig. 2c) and C (Fig. 3c), but coupled with an order of magnitude change in $E_{\mathrm{g}}$, leads to a difference of two orders of magnitude in $\eta_{\mathrm{e}}-\mathrm{h}$. The change in $\eta_{\mathrm{e}-\mathrm{h}}$ between Devices $\mathrm{A}$ and $\mathrm{C}$ can thus be predominantly ascribed to their different $E_{\mathrm{g}}$ 's. This inverse dependence of $\eta_{\mathrm{e}-\mathrm{h}}$ on $E_{\mathrm{g}}$ can be understood by inspecting Fig. 2a,b. The relative $e-h$ barrier height asymmetry decreases rapidly as $E_{\mathrm{g}}$ increases and becomes larger than the doping $\Delta E_{\mathrm{F}, 1}$ induced by the gold gates.

As the values of $E_{\mathrm{C}}$ and $\phi_{B}$ in our SWCNT-QD transistors are large compared with $k_{\mathrm{B}} T_{\text {room }}$, we can envision making use of the $e-h$ transport asymmetry in room temperature $\mathrm{QD}$ devices. Figure $4 \mathrm{~d}$ shows $I-V_{\mathrm{B}}-V_{\mathrm{G}}$ data for Device B at $295 \mathrm{~K}$, with a superimposed $I-V_{\mathrm{G}}$ trace taken at $V_{\mathrm{B}}=10 \mathrm{mV}$. The SEM image of Device B in Fig. 1c shows a channel length of only $14 \mathrm{~nm}$ and transport data a bandgap of $270 \mathrm{meV}$ (Supplementary Note 2). During the warm up from $1.3 \mathrm{~K}$ to room temperature, Device $\mathrm{B}$ was exposed to oxygen (Supplementary Note 1) leading to p-doped SWCNT contact sections (Supplementary Fig. 2). In Fig. $4 \mathrm{~d}$, remnants of conductance oscillations are visible when the channel is $p$-doped. The spacing of these oscillations matches the $L_{\mathrm{G}}$ measured at low temperature and suggests the formation of a $\mathrm{QD}$ with an $E_{\mathrm{C}}^{\mathrm{h}} \sim 25 \mathrm{meV}$. The data in Supplementary Fig. 2 are consistent with $E_{\mathrm{C}}^{\mathrm{e}}>E_{\mathrm{C}}^{\mathrm{h}}$ at $295 \mathrm{~K}$ and we expect the $e-h$ asymmetry to survive at room temperature.

In summary, we used suspended annealed gold films as local gates to create $e-h$ asymmetric suspended SWCNT-QD transistors (Fig. 1e). Using transport measurements, we showed that the gold gates permit ballistic (coherent) charge transport in the underlying SWCNT sections and create nanometre-sharp tunnel barriers at the edge of the SWCNT channels. These 
a

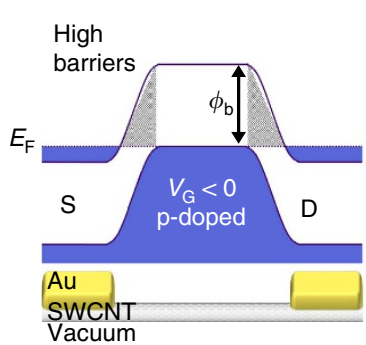

b

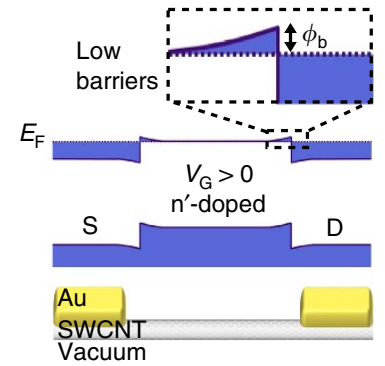

d

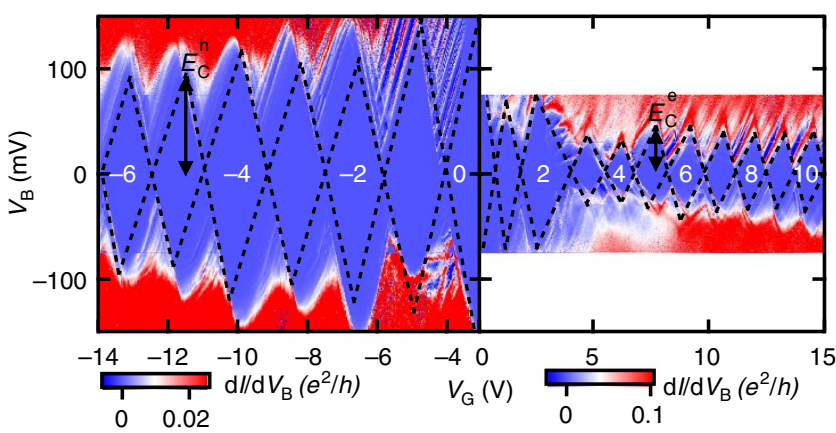

C

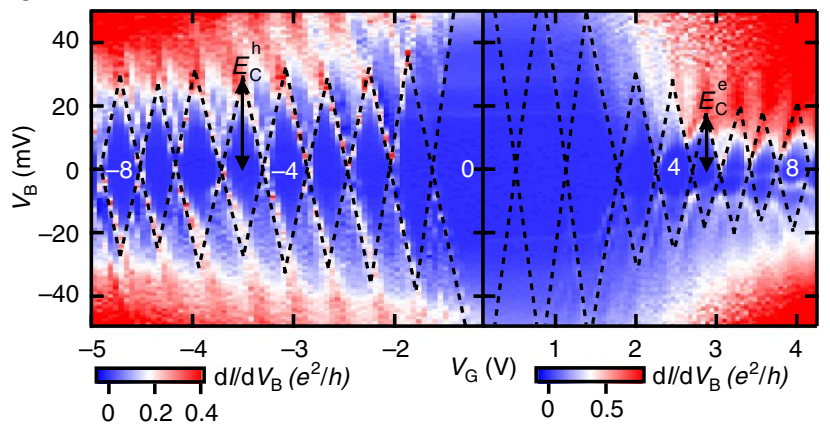

e

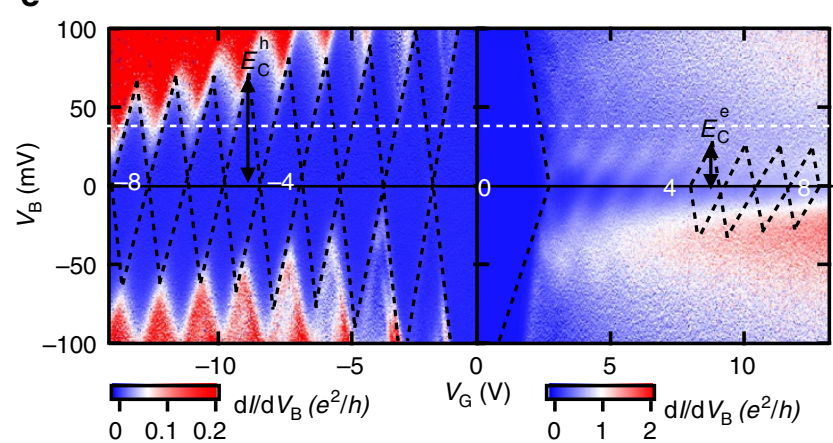

$f$

h

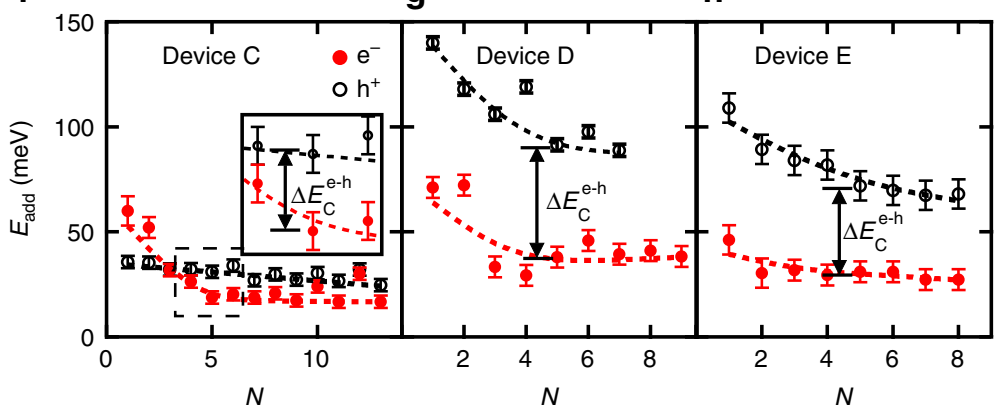

i

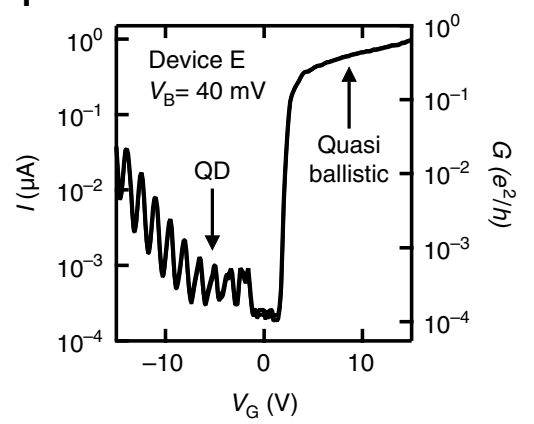

Figure 3 | Giant $\mathbf{e}-\boldsymbol{h}$ charging energy asymmetry in $\mathbf{2 0 0} \mathbf{m e V}$ band gap nanotube QDs. (a,b) Schematics of the junctions between the $n$-doped nanotube contacts and naked tube channel in Device $C, E_{g}=190 \mathrm{meV}$. (a) When the channel is $p$-doped, the band-to-band tunnel barriers (shaded triangles) have a height $\phi_{\mathrm{B}}=190 \mathrm{meV}$. (b) When the channel is $n$-doped, the tunnel barriers are much smaller, $\phi_{\mathrm{B}} \sim 12 \mathrm{meV}$ (Supplementary Note 4 ). (c-e) $d l / d V_{B}-V_{B}-V_{G}$ transport data for Devices $C, D$ and $E$, respectively $(T=4.0,1.3$ and $50 \mathrm{~K}$ ). In all devices, clear Coulomb diamonds are visible for both hole and electron channel dopings and show the formation of a single QD in the channel. The charging energies, that is, heights of the odd-labelled diamonds, are much larger for holes than for electrons due to the tunnel barrier asymmetry. (f-h) Extracted addition energy, $E_{\text {add }}=$ height of diamonds, versus charge number, $N$, for both holes (open black data) and electrons (filled red data) in Devices $C, D$ and $E$. The data for odd $N$, for which $E_{\text {add }}=E_{C}$ are interpolated with dashed lines. The charging energies decrease with increasing $N$ and a clear offset, $\Delta E_{C}^{e-h}$ is visible between hole and electron data. The value of $\Delta E_{C}^{e^{-h}}$ becomes roughly constant at large $N$. The errors bars represent the uncertainty in the extracted $E_{\text {add }}$ and stem from the limited resolution of the Coulomb diamond heights in c-e. (i) $I-V_{G}$ data for Device $E$ at $V_{B}=40 \mathrm{meV}$ (along white dashed line in e).

barriers were of vastly different heights whether a channel was hole or electron doped. This produced a giant $e-h$ transport asymmetry in the five SWCNT transistors studied. We used this asymmetry to create two types of SWCNT quantum devices with dual functionality. We first showed a low- $E_{\mathrm{g}}$ SWCNT device where a small gate voltage could switch the device from being a $330 \mathrm{~nm}$-long quantum bus, under electron doping, to a $102 \mathrm{~nm}$ long QD, for hole doping. Second, we reported four devices with $E_{\mathrm{g}} \gtrsim 200 \mathrm{meV}$ where a small $V_{\mathrm{G}}$ could switch the QD between two charging energies whose values differed by a factor up to 2.6, or switch the transport from QD to nearly ballistic. The size of the $e-h$ transport asymmetry in these devices scaled inversely with the length of the channel and the band gap of the tube. In a $14 \mathrm{~nm}$-long channel device, we measured low-temperature charging energies for holes and electrons exceeding 100 and $50 \mathrm{meV}$, respectively, which suggests that the $e-h$ asymmetry could survive in room temperature devices. Nanotube transistors with a giant $e-h$ transport asymmetry could find applications in exploring the physics of near molecular size SWCNT-NEMS ${ }^{46}$, to shrink down SWCNT qubits ${ }^{12,47}$ and to create SWCNT THz detectors ${ }^{15}$ or gate programmable transistors ${ }^{16}$.

\section{Methods}

Lithography. We use $e$-beam lithography to define bow-tie-shaped breakjunctions in a poly(methyl methacrylate) bilayer on SWCNTs deposited on a $\mathrm{SiO}_{2} / \mathrm{Si}$ substrate. We then evaporate a $40 \mathrm{~nm}$-thick gold film (no adhesion layer) to define the gold junctions. We use a wet buffered oxide etch to freely suspend the central portion of the gold breakjunctions as shown in Fig. 1a.

Electromigration. The red data in Fig. 1b show the first stage of EM where we narrow down the central bowtie-shaped gold junctions. To do so, we ramp up a bias voltage, $V_{\mathrm{B}}$, across the device and monitor the current, $I$. The bias is applied to the source electrode and the drain is grounded. As the resistance, $R=V_{B} / I$, 
$\mathbf{a}$

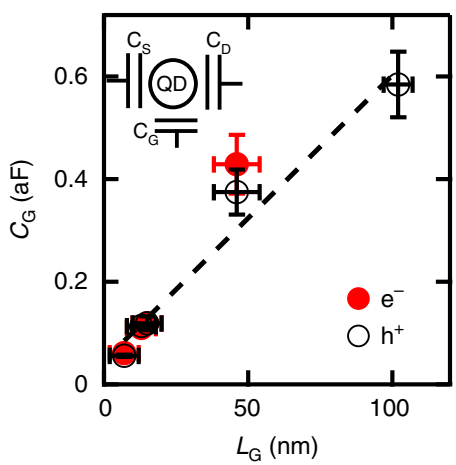

C

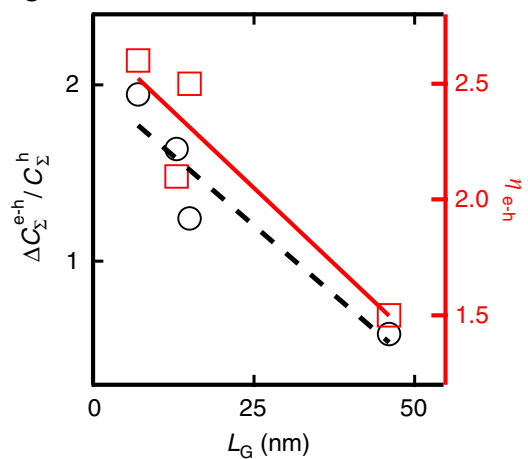

b

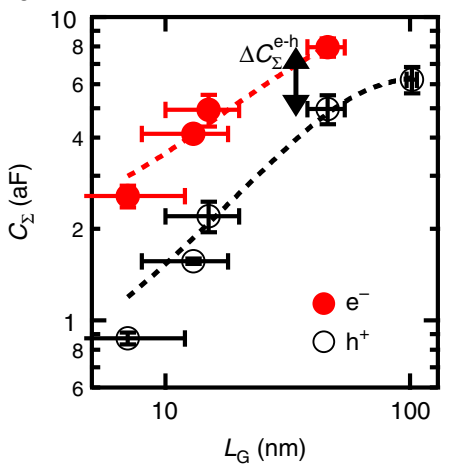

d

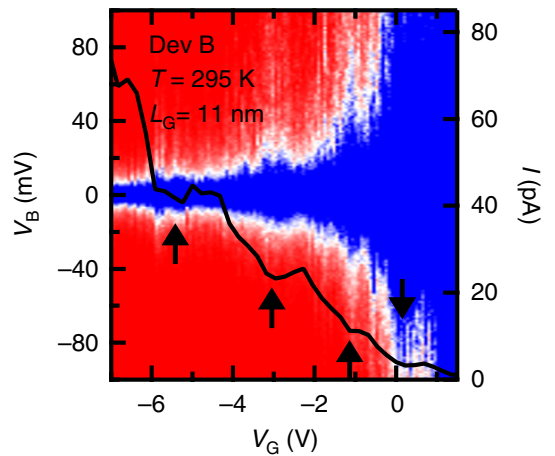

Figure 4 | Origin of the $\mathbf{e}-\boldsymbol{h}$ charging energy asymmetry. (a) The gate-to-dot capacitance, $C_{G}$, in our devices is $e-h$ symmetric and scales linearly with $L_{G}$. The inset cartoon of the $Q D$ devices shows the lumped element interpretation of $C_{G}, C_{S}$ and $C_{D}$. The error bars represent the uncertainty in the widths of the Coulomb diamonds in the transport data of Fig. 3 and Supplementary Fig. 3. (b) The total QD capacitance $C_{\Sigma}$ increases approximately linearly as a function of $L_{G}$ in our devices. A clear capacitance offset $\Delta C_{\Sigma}^{e-h}$ is visible between the hole and electron data due to the e- $h$ tunnel barrier asymmetry. This offset is at the origin of the $e-h$ charging energy asymmetry. The error bars represent the uncertainty in the total QD capacitance stemming from the uncertainties in the slopes of the Coulomb diamonds in the transport data of Fig. 3 and Supplementary Fig. 3. (c) The relative e-h capacitance asymmetry $\Delta C_{\Sigma}^{\mathrm{e}-\mathrm{h}} / C_{\Sigma}^{\mathrm{h}}$ decreases with increasing $L_{\mathrm{G}}$ and explains why $\eta_{\mathrm{e}-\mathrm{h}}=E_{C}^{\mathrm{h}} / E_{C}^{\mathrm{e}}$ also decreases with increasing $L_{\mathrm{G}}$. The dashed and solid lines are linear fits of the data. (d) $I-V_{B}-V_{G}$ data in Device $B$ at $295 \mathrm{~K}$ with a superimposed $I-V_{G}$ data cut, $V_{B}=10 \mathrm{mV}$. The spacing of the conductance oscillation (black arrows) gives $L_{G} \approx 11 \mathrm{~nm}$.

increases, a feedback circuit rapidly ramps down $V_{\mathrm{B}}$ to avoid an avalanche breaking of the junction. We repeat the feedback controlled-EM process iteratively to gradually narrow down the junction (increase $R$ ) and these successive voltage ramps are visible in the inset of Fig. 1b. We learned previously that using a continuous $V_{\mathrm{B}}$ ramp to break a gold junction gives a central junction gap which is proportional to the initial resistance value of the junction ${ }^{5,21,22}$. Thus, we stop the narrowing of the gold junction at a desired initial resistance to achieve a target channel length. We then proceed to step two of the EM (blue data), where the junction is broken with a single continuous voltage ramp.

Band gap measurement. To determine the band gaps of the tubes in our devices, we use the data at the charge neutrality point $N=0$, corresponding to the largest blockade diamond in Figs $2 \mathrm{c}$ and $3 \mathrm{c}-\mathrm{e}$, and Supplementary Fig. 3. The band gap is given by $E_{\mathrm{g}}=E_{\text {add }^{\mathrm{N}=0}}-E_{\mathrm{C}^{\mathrm{N}=0}}-\Delta$ where $_{\mathrm{add}^{\mathrm{N}=0}}$ is the height of the $N=0$ diamond, $E_{\mathrm{C}}$ is the charging energy and $\Delta$ is the single-particle energy spacing. See Supplementary Note 2 for details.

Data availability. The data that support the findings of this study are available from the corresponding author upon request.

\section{References}

1. Jarillo-Herrero, P., Sapmaz, S., Dekker, C., Kouwenhoven, L. P. \& van der Zant, H. S. J. Electron-hole symmetry in a semiconducting carbon nanotube quantum dot. Nature 429, 389-392 (2004).

2. Laird, E. A. et al. Quantum transport in carbon nanotubes. Rev. Mod. Phys. 87, 703-764 (2015).

3. Steele, G. A., Gotz, G. \& Kouwenhoven, L. P. Tunable few-electron double quantum dots and Klein tunnelling in ultraclean carbon nanotubes. Nat. Nanotechnol. 4, 363-367 (2009).

4. Jung, M. et al. Ultraclean single, double, and triple carbon nanotube quantum dots with recessed re bottom gates. Nano Lett. 13, 4522-4526 (2013).
5. Island, J. O., Tayari, V., McRae, A. C. \& Champagne, A. R. Few-hundred GHz carbon nanotube nanoelectromechanical systems (NEMS). Nano Lett. 12, 4564-4569 (2012).

6. Chaste, J. et al. A nanomechanical mass sensor with yoctogram resolution. Nat. Nanotechnol. 7, 301-304 (2012).

7. Benyamini, A., Hamo, A., Kusminskiy, S. V., von Oppen, F. \& Ilani, S. Real-space tailoring of the electron-phonon coupling in ultraclean nanotube mechanical resonators. Nat. Phys. 10, 151-156 (2014).

8. Moser, J., Eichler, A., Guttinger, J., Dykman, M. I. \& Bachtold, A. Nanotube mechanical resonators with quality factors of up to 5 million. Nat. Nanotechnol. 9, 1007-1011 (2014).

9. Weber, P. et al. Switchable coupling of vibrations to two-electron carbonnanotube quantum dot states. Nano Lett. 15, 4417-4422 (2015).

10. Kuemmeth, F., Ilani, S., Ralph, D. C. \& McEuen, P. L. Coupling of spin and orbital motion of electrons in carbon nanotubes. Nature 452, 448-452 (2008).

11. Pei, F., Laird, E. A., Steele, G. A. \& Kouwenhoven, L. P. Valley-spin blockade and spin resonance in carbon nanotubes. Nat. Nanotechnol. 7, 630-634 (2012).

12. Laird, E. A., Pei, F. \& Kouwenhoven, L. P. A valley-spin qubit in a carbon nanotube. Nat. Nanotechnol. 8, 565-568 (2013).

13. Bergfield, J. P. \& Ratner, M. A. Forty years of molecular electronics: nonequilibrium heat and charge transport at the nanoscale. Phys. Stat. Solid. B 250, 2249-2266 (2013).

14. Zonda, M., Pokorny, V., Janis, V. \& Novotny, T. Perturbation theory for an Anderson quantum dot asymmetrically attached to two superconducting leads. Phys. Rev. B 93, 024523-1-024523-18 (2016).

15. Rinzan, M., Jenkins, G., Drew, H. D., Shafranjuk, S. \& Barbara, P. Carbon nanotube quantum dots as highly sensitive terahertz-cooled spectrometers. Nano Lett. 12, 3097-3100 (2012).

16. Yoo, H. et al. Reconfigurable complementary logic circuits with ambipolar organic transistors. Sci. Rep. 6, 35585-1-35585-11 (2016).

17. Shulaker, M. M. et al. Carbon nanotube computer. Nature 501, 526-530 (2013) 
18. Wu, C. C., Liu, C. H. \& Zhong, Z. H. One-step direct transfer of pristine singlewalled carbon nanotubes for functional nanoelectronics. Nano Lett. 10, 1032-1036 (2010)

19. Cao, J., Wang, Q., Rolandi, M. \& Dai, H. J. Aharonov-bohm interference and beating in single-walled carbon-nanotube interferometers. Phys. Rev. Lett. 93, 216803-216804 (2004).

20. LeRoy, B. J., Lemay, S. G., Kong, J. \& Dekker, C. Electrical generation and absorption of phonons in carbon nanotubes. Nature 432, 371-374 (2004).

21. Island, J. O., Tayari, V., Yigen, S., McRae, A. C. \& Champagne, A. R. Ultra-short suspended single-wall carbon nanotube transistors. Appl. Phys. Lett. 99, 243106-1-243106-4 (2011).

22. Tayari, V. et al. Tailoring $10 \mathrm{~nm}$ scale suspended graphene junctions and quantum dots. Nano Lett. 15, 114-119 (2015).

23. Giovannetti, G. et al. Doping graphene with metal contacts. Phys. Rev. Lett. 101, 026803-026804 (2008).

24. Cui, X. D., Freitag, M., Martel, R., Brus, L. \& Avouris, P. Controlling energylevel alignments at carbon nanotube/Au contacts. Nano Lett. 3, 783-787 (2003).

25. Hasegawa, M. \& Nishidate, K. Transfer doping of a metallic carbon nanotube and grapheme on metal surfaces. Phys. Rev. B 84, 155435-1-155435-11 (2011).

26. Heinze, S. et al. Carbon Nanotubes as Schottky Barrier Transistors. Phys. Rev. Lett. 89, 106801-106804 (2002).

27. Jorio, A. et al. Structural (n,m) determination of isolated single-wall carbon nanotubes by resonant Raman scattering. Phys. Rev. Lett. 86, 1118-1121 (2001).

28. Liu, K. H. et al. High-throughput optical imaging and spectroscopy of individual carbon nanotubes in devices. Nat. Nanotechnol. 8, 917-922 (2013).

29. Knoch, J. \& Appenzeller, J. Tunneling phenomena in carbon nanotube fieldeffect transistors. Phys. Stat. Solid. A 205, 679-694 (2008).

30. Tersoff, J. Contact resistance of carbon nanotubes. Appl. Phys. Lett. 74, 2122-2124 (1999)

31. Sundaram, R. S. et al. The graphene-gold interface and its implications for nanoelectronics. Nano Lett. 11, 3833-3837 (2011).

32. Liang, W. et al. Fabry-Perot interference in a nanotube electron waveguide. Nature 411, 665-669 (2001).

33. Makarovski, A., Liu, J. \& Finkelstein, G. Evolution of transport regimes in carbon nanotube quantum dots. Phys. Rev. Lett. 99, 066801-066804 (2007).

34. Jorgensen, H., Grove-Rasmussen, K., Flensberg, K. \& Lindelof, P. E. Critical and excess current through an open quantum dot: temperature and magnetic-field dependence. Phys. Rev. B 79, 155441-155446 (2009).

35. Khomyakov, P. A. et al. First-principles study of the interaction and charge transfer between graphene and metals. Phys. Rev. B 79, 195425-1-195425-12 (2009).

36. Chaves, F. A., Jimenez, D., Cummings, A. W. \& Roche, S. Physical model of the contact resistivity of metal-graphene junctions. J. Appl. Phys. 115, 164513-164518 (2014).

37. Appenzeller, J., Lin, Y. M., Knoch, J. \& Avouris, P. Band-to-band tunneling in carbonnanotube field-effect transistors. Phys. Rev. Lett. 93, 196805-1-196805-4 (2004).

38. Schmidt, M. Fabrication, Characterization and Simulation of Band-to-Band Tunneling Field-Eect Transistors Based on Silicon-Germanium. (Ph.D. thesisRWTH Aachen University, 2013).

39. Lu, W., Wang, D. \& Chen, L. W. Near-static dielectric polarization of individual carbon nanotubes. Nano Lett. 7, 2729-2733 (2007).

40. Nemec, N., Tomanek, D. \& Cuniberti, G. Contact dependence of carrier injection in carbon nanotubes: an ab initio study. Phys. Rev. Lett. 96, 076802-076804 (2006).
41. Franklin, A. D. et al. Sub-10 nm carbon nanotube transistor. Nano Lett. 12, 758-762 (2012).

42. Kuemmeth, F., Churchill, H. O. H., Herring, P. K. \& Marcus, C. M. Carbon nanotubes for coherent spintronics. Mater. Today 13, 18-26 (2010).

43. Pecker, S. et al. Observation and spectroscopy of a two-electron Wigner molecule in an ultraclean carbon nanotube. Nat. Phys. 9, 576-581 (2013).

44. Yang, Y. et al. Coherent nonlocal transport in quantum wires with strongly coupled electrodes. Phys. Rev. B 87, 045403-045405 (2013).

45. Grove-Rasmussen, K., Jorgensen, H. I. \& Lindelof, P. E. Fabry-Perot interference, Kondo effect and Coulomb blockade in carbon nanotubes. Phys. E 40, 92-98 (2007).

46. Cornaglia, P. S., Ness, H. \& Grempel, D. R. Many-body effects on the transport properties of single-molecule devices. Phys. Rev. Lett. 93, 147201-147204 (2004).

47. Wunsch, B. Few-electron physics in a nanotube quantum dot with spin-orbit coupling. Phys. Rev. B 79, 235408-1-235408-6 (2009).

\section{Acknowledgements}

This work was supported by NSERC (Canada), CFI (Canada) and Concordia University We acknowledge usage of the QNI (Quebec Nano Infrastructure) cleanroom network.

\section{Author contributions}

A.C.M. led all steps of the fabrication of the samples and acquired all the data reported A.R.C. conceived and supervised the experiment. A.C.M. and A.R.C. analysed the data V.T. and J.M.P. contributed to the sample fabrication and the development of the experimental methods. A.R.C. and A.C.M. wrote the manuscript. All authors discussed the results and the manuscript.

\section{Additional information}

Supplementary Information accompanies this paper at http://www.nature.com/ naturecommunications

Competing interests: The authors declare no competing financial interests.

Reprints and permission information is available online at http://npg.nature.com/ reprintsandpermissions/

How to cite this article: McRae, A. C. et al. Giant electron-hole transport asymmetry in ultra-short quantum transistors. Nat. Commun. 8, 15491 doi: 10.1038/ncomms15491 (2017).

Publisher's note: Springer Nature remains neutral with regard to jurisdictional claims in published maps and institutional affiliations

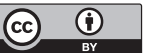

This work is licensed under a Creative Commons Attribution 4.0 International License. The images or other third party material in this article are included in the article's Creative Commons license, unless indicated otherwise in the credit line; if the material is not included under the Creative Commons license, users will need to obtain permission from the license holder to reproduce the material. To view a copy of this license, visit http://creativecommons.org/licenses/by/4.0/

C) The Author(s) 2017 